\title{
Verrucous Hemangioma Mimicking Melanoma in an Elderly Man
}

\author{
L. Pérez-Varela ${ }^{1}$, J. Del Pozo ${ }^{1}$, F. Pineyro ${ }^{1}$, F. Sacristán ${ }^{2}$, C. Peña ${ }^{1}$, B. Fernández-Jorge ${ }^{1}$, \\ R. Rodríguez-Lojo ${ }^{1}$, A. Wong ${ }^{3}$
}

${ }^{1}$ Department of Dermatology, CHU, A Coruña, Spain; ${ }^{2}$ Department of Pathology, CHU, A Coruña, Spain; ${ }^{3}$ Department of Pathology, Yale University School of Medicine, New Haven, USA.

E-mail: jesus.del.pozo.losada@sergas.es

Received September $10^{\text {th }}, 2011$; revised October $28^{\text {th }}$, 2011; accepted November $9^{\text {th }}, 2011$.

\begin{abstract}
Verrucous hemangioma $(\mathrm{VH})$ is an uncommon, congenital, vascular anomaly that resembles angiokeratoma but it encompasses distinctive clinicopathological features. The differential diagnosis of this entity included all the hyperkeratotic vascular tumors and malformations and, especially in localized cases, pigmented lesions. VH lesions initially present at birth, and therefore, the diagnosis in the elderly may be difficult. We present a 77 year-old patient with a birth lesion on the back of his calf who came to the clinic with a two year history of enlargement of this lesion following trauma. We performed an excisional biopsy because clinical appearance simulated melanoma arising from a congenital nevus. Nevertheless, the histopathological examination revealed a verrucous hemangioma. A diagnosis of VH should be considered in erythematous papules, nodules, and plaques, especially those that are hyperkeratotic, present at birth, located on the lower extremities, and show growth after trauma or infection.
\end{abstract}

Keywords: Angiokeratoma, Melanoma, Verrucous Hemangioma

\section{Introduction}

Despite the overall success of the ISSVA classification system, there are some vascular lesions in which the clinical and histopathologic findings are insufficient to determine whether a lesion is a tumor or a malformation. Recent evidence suggests that some of these lesions may share histologic features of both hemangiomas and malformations, thereby causing nosologic confusion [1,2]. Verrucous hemangioma ( $\mathrm{VH})$ is such an example.

Verrucous hemangioma ( $\mathrm{VH}$ ) is an uncommon, congenital, localized, vascular anomaly that clinically resembles an angiokeratoma but it presents with distinctive clinicopathological features.

The classification of $\mathrm{VH}$ as a vascular malformation is supported by its presence at birth, anatomic distribution, proportionate growth and usually negative stain with WT1 (Wilms tumor-1 gene) [3]. However, the thick vascular walls with multilamellated basement membranes, relative uniformity of channel size, occasional lobularity, and focal GLUT1 positivity are features that invite comparison with infantile hemangioma in its involutive phase [1].
Another controversial fact in $\mathrm{VH}$ is the name. The ending "oma" suggests a tumor, but VH is not clearly a hemangioma. As a result, this entity was known by several names. Some recently proposed names include hemangioma unilateralis neviforme, unilateral verrucous hemangioma, angiokeratoma circumscriptum neviforme, nevus vascularis unius lateralis, keratotic hemangioma, nevus angiokeratoticus, nevus keratoangiomatosus, papulous angiokeratoma and verrucous lymphovascular malformation [2]. Historical nomenclature for hyperkeratotic vascular lesions confounds our understanding of VH. For example, Imperial and Helwing [4] designated $\mathrm{VH}$ as a vascular malformation, but in the same paper they also described VH as a "structural variant of capillary or cavernous hemangioma". Mankani and Dufresne [5] reexamined the histopathology of $\mathrm{VH}$ and, using the International Society for the Study of Vascular Anomalies (ISSVA) binary system, recommended a new name, verrucous [vascular] malformation.

VH clinically presents as circumscribed, warty papules or plaques, or as lesions arranged in linear or serpiginous configuration. Lesions are often located on the lower extremities but other localizations, such as the trunk and 
upper extremities have been described. Usually the verrucous component increases with time and occasionally bleeds [6].

Histopathologically, VH is characterized by dilated capillaries and large cavernous, endothelial-lined, bloodfilled spaces extending well into the reticular dermis and subcutaneous fat and with hyperkeratosis [7].

The course of VH is generally characterized by a slow, superficial spreading growth pattern. They have no tendency for spontaneous involution, often develop of satellite lesions [8], and recur after excision (30\% of cases), especially when the lesions are larger than $2 \mathrm{~cm}$ in diameter [7].

Misdiagnosis of this entity is common. We present a case of VH diagnosed in the elderly with clinical suspicion of malignant melanoma.

\section{Case Report}

A 77-year-old man presented to our outpatient clinic with a birth lesion on the back of his calf with concerns of the growth in the last years following trauma. Dermatologic examination showed a verrucous, hyperkeratotic, wellcircumscribed, hemorrhagic crusted, solitary, heterogeneous dark, excrescent nodule with a diameter of $2 \times 2$ $\mathrm{cm}$. The underlying skin was infiltrated (Figure 1). We decided to perform an excisional biopsy because the clinical appearance simulated melanoma arising on congenital nevus. During the surgery, we observed satellite lesions, so we decided to make a bigger incision, thinking they were in-transit metastasis. A laminar graft was performed to close the surgical defect. The histopathologic examination showed a lesion with irregular papillary acanthosis, parakeratosis, and hyperkeratosis in the epidermis. Abnormal vessels were located in the dermis and subcutis, not closely apposed and were separated by fibrous tissue in the dermis and fibrous or adipose tissue in the subcutis (Figure 2). The vessels were round and thin-walled. Endothelial cells were flat and inconspicuous. In some areas, a lobular appearance was observed because the vessels occupied an entire adipocytic lobule.

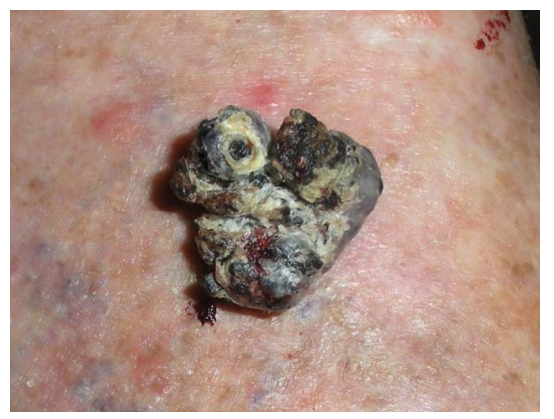

Figure 1. Hyperkeratotic, well-circumscribed, excrescent nodule on the back of the calf.
The more superficial vessels often contained blood, whereas the deeper vessels contained blood or were empty. The vessels had focal endothelial immunopositivity for GLUT1 (Figure 3) and were also positive for WT-1.

A diagnosis of verrucous hemangioma was made. After a follow-up of several years, no relapse of lesion was detected.

\section{Discusion}

Hyperkeratotic vascular lesions are a heterogeneous group and include vascular malformations such as capillary malformations (angiokeratomas including angiokeratoma circumscriptum naeviformis), capillary-lymphatic malformations, capillary-venous malformations, capillaryvenous-lymphatic malformations (Klippel-Trenaunay syndrome), lymphatic malformations, venous malformations (Cerebral cavernous malformation, known to be caused by KRIT1 mutations, are associated (12\%) with

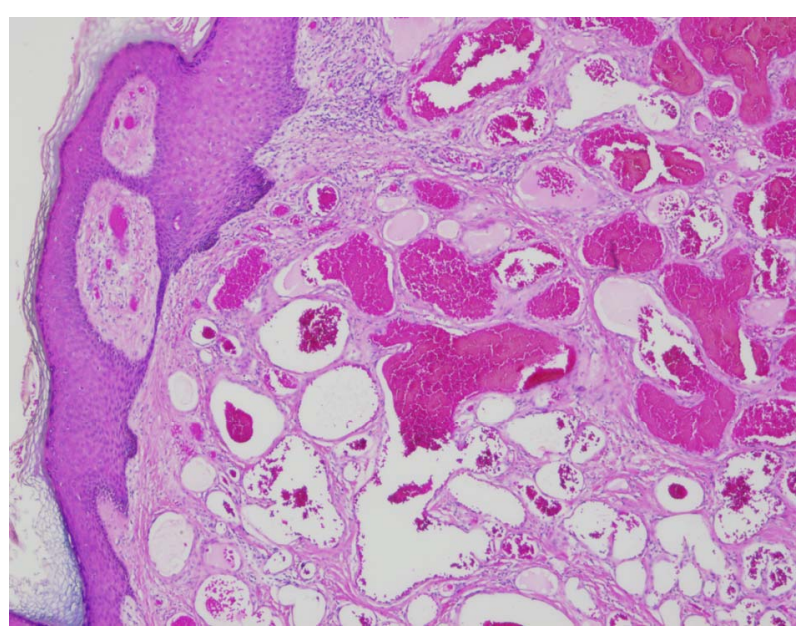

Figure 2. Abnormal thin-walled vessels in the dermis, containing blood (H-E, 200×).

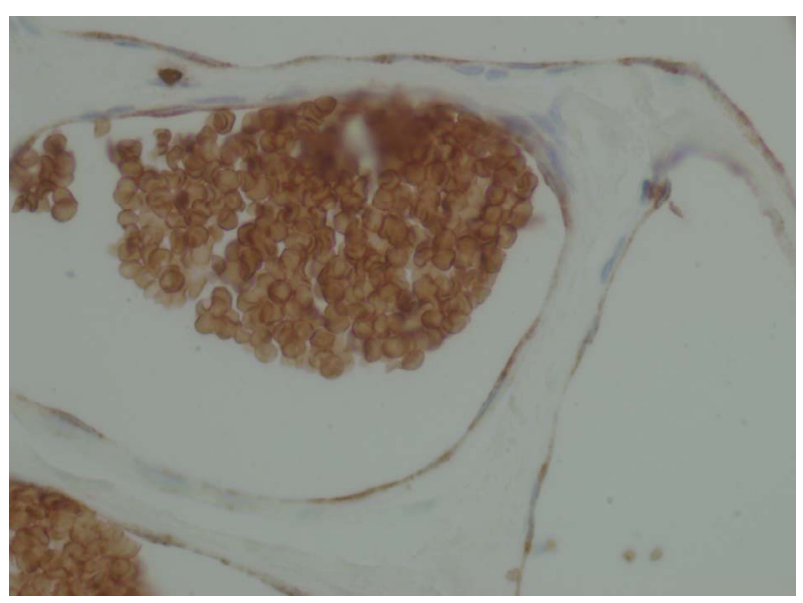

Figure 3. Endothelial immunopositivity for GLUT1. The red blood cells were also positive $(400 \times)$. 
hyperkeratotic cutaneous CVMs that resemble $\mathrm{VH}$ ), traumatic arteriovenous fistula and verrucous haemangioma. In addition, other hyperkeratotic lesions different of vascular anomalies that clinically must be differentiated from VH are verruca and pigmented tumours [9].

Angiokeratomas are rare verrucous vascular lesions that appear as dark red papules and plaques arranged either discretely or in clusters. The exact mechanism for their development is not known, although congenital cause, pregnancy, chilblains, trauma, and tissue asphyxia are given as causal factors [10]. The basic pathological process is dilatation of the papillary capillaries with reactive epidermal changes of hyperkeratosis and acanthosis as a means of preventing further dilatation and rupture. If thrombosis occurs within an angiokeratoma, the lesion may clinically mimic malignant melanoma [11,12]. It could be because the accompanying inflammatory reaction causes that $\mathrm{VH}$ loses its normal vascular appearance and acquires a purplish-dark color, so this lesion could be confused with a melanocytic lesion. VH are frequently mistakenly classified as a variety of angiokeratoma. However, in contrast to angiokeratoma, the vascular spaces in $\mathrm{VH}$ also involve the lower dermis and subcutaneous tissues and therefore the lesion may recur if inadequately excise. A diagnosis of $\mathrm{VH}$ should be highly considered in lesions with a hyperkeratotic nodular appearance located on the lower extremities. VH do not resolve spontaneously and have a tendency to relapse. VH are best treated by excision while still small, as they enlarge with body growth and do no regress spontaneously. VH usually require a large, deep excision, in contrast with angiokeratomas, which respond to various means of therapy such as cryosurgery, electrocautery, and laser therapy [13].

Angiokeratoma circumscriptum is classified among the hypertrophic angio-producing tumors (telangectasias), characterized by a single vascular dilatation without cellular proliferation, while, verrucous hemangioma is considered among the angio-producing hyperplastic tumors, characterized by proliferation of mesenchymal cells tending to the formation of capillaries [14,15]. The correct diagnosis is made by the histopathological features, although a correct clinical suspicion is important in order to obtain an adequate deep biopsy specimen for ruling out verrucous hemangioma.

Clinical aspect of lesions, radiological studies, and histopathological features help us to differentiated VH to other vascular malformations with hyperkeratotic aspect.

The violaceous to black aspect of these lesions usually induce clinical confusion of $\mathrm{VH}$ with melanocytic lesions, mainly in localized and/or old cases of $\mathrm{VH}$ as our case. In addition, $\mathrm{VH}$ commonly increase in size after a traumatic event as in our case, and in these cases, a malignant melanoma must be excluded. Dermoscopy may help to differentiate these lesions and increases the percentage of correct diagnosis [16].

Surgery with adequate margins was performed in our patient and the result was good, with no recurrence after eight years.

A diagnosis of VH should be considered in erythematous papules, nodules, and plaques, especially those that are hyperkeratotic, present at birth, located on the lower extremities, and show growth after trauma or infection. These lesions must be confirmed by histopathological examination.

\section{REFERENCES}

[1] L. B. Tennant, J. B. Mulliken, A. R. Perez-Atayde and H. P. Kozakewich, "Verrucous Hemangioma Revisited," Pediatric Dermatology, Vol. 23, No. 3, 2006, pp. 208-215. doi:10.1111/j.1525-1470.2006.00219.x

[2] A. Brown, S. Warren, H. W. Losken and D. S. Morrell, "Verrucous Lymphovascular Malformation versus Verrucous Hemangioma: Controversial Nomenclature,” Cutis, Vol. 81, No. 5, 2008, pp. 390-396.

[3] R. Al Dhaybi, J. Powell, C. McCuaig and V. Kokta, "Differentiation of Vascular Tumors from Vascular Malformations by Expression of Wilms Tumor 1 Gene: Evaluation of 126 Cases," Journal of the American Academy of Dermatology, Vol. 63, No. 6, 2010, pp. 1052-1057. doi:10.1016/j.jaad.2009.12.017

[4] R. Imperial and E. B. Helwig, "Verrucous Hemangioma. A Clinicopathologic Study of 21 Cases," Archives of Dermatology, Vol. 96, No. 3, 1967, pp. 247-253. doi:10.1001/archderm.1967.01610030025004

[5] M. H. Mankani and C. R. Dufresne, "Verrucous Malformations: Their Presentation and Management," Annals of Plastic Surgery, Vol. 45, No. 1, 2000, pp. 31-36. doi:10.1097/00000637-200045010-00006

[6] I. Lara-Corrales, G. R. Somers and N. Ho, "Verrucous Hemangioma: A Challenging Vascular Lesion,” Journal of Cutaneous Medicine and Surgery, Vol. 14, No. 3, 2010, pp. 144-146.

[7] L. Calduch, C. Ortega, V. Navarro, E. Martínez, I. Molina, and E. Jordá, "Verrucous Hemangioma: Report of Two Cases and Review of the Literature," Pediatric Dermatology, Vol. 17, No. 3, 2000, pp. 213-217. doi:10.1046/j.1525-1470.2000.01755.x

[8] M. Koc, M. Kavala, E. Kocatürk, E. Zemheri, I. Zindanci, S. Sudogan and E. Kural, "An Unusual Vascular Tumor: Verrucous Hemangioma,” Dermatology Online Journal, Vol. 15, No. 11, 2009 , p. 7.

[9] V. K. Jain, K. Aggarwal and S. Jain, "Linear Verrucous Hemangioma on the Leg," Indian Journal of Dermatology, Venereology and Leprology, Vol. 74, No. 6, 2008, pp. 656-658. doi:10.4103/0378-6323.45119

[10] R. Loria, J. Derbes and J. D. Kerafchuck, "Keratotic Hemangiomas,” Archives of Dermatology , Vol. 74, 1956, pp. 437-438. 
[11] L. Goldman, S. H. Gibson and D. F. Richfield, "Thrombotic Angiokeratoma Circumscriptum Simulating Melanoma,” Archives of Dermatology, Vol. 117, No. 3, 1981, pp. 138-139.

doi:10.1001/archderm.1981.01650030016009

[12] F. De Dulanto, M. A. Moreno, F. C. Martinez and R. N. Sintes, "Solitary Black Angiokeratoma and Verrucous Hemangioma. Problems and Transcendency of Differential Diagnosis with Malignant Melanoma. Treatment," Actas Dermosifiliogr, Vol. 66, No. 7-8, 1975, pp. 367-386.

[13] P. I. Schiller and P. H. Itin, “Angiokeratomas: An Update,” Dermatology, Vol. 193, No. 4, 1996, pp. 275-282. doi:10.1159/000246270

[14] A. Rossi, M. Bozzi and E. Barra, "Verrucous Hemangioma and Angiokeratoma Circumscriptum: Clinical and
Histological Differential Characteristics,” Journal of Dermatologic Surgery \& Oncology, Vol. 15, No. 1, 1989, pp. 88-91.

[15] J. Del Pozo and E. Fonseca, “Angiokeratoma Circumscriptum Naeviforme: Succesfull Treatment with Carbon Dioxide Laser Vaporization,” Dermatologic Surgery, Vol. 31, No. 2, 2005, pp. 232-236. doi:10.1111/j.1524-4725.2005.31048

[16] M. T. Sahin, A. Türel-Ermertcan, S. Oztürkcan and P. Türkdogan, “Thrombosed Solitary Angiokeratoma of Mibelli Simulating Malignant Melanoma: The Importance of Dermoscopy in Differential Diagnosis," Journal of the European Academy of Dermatology and Venereology, Vol. 20, No. 1, 2006, pp. 102-104. doi:10.1111/j.1468-3083.2005.01299.x 